\title{
Pancreatic Neuroendocrine Tumors Complicated by Sinistral Portal Hypertension: Insights into Pathogenesis
}

\author{
Terence N. Moyana, ${ }^{1 *}$ D. Blair Macdonald, ${ }^{2}$ Guillaume Martel, ${ }^{3}$ Sergey Pyatibrat, ${ }^{1}$ Goo Lee, ${ }^{1}$ and Mario Capitano
}

\begin{abstract}
Purpose: To investigate the association between pancreatic neuroendocrine tumors (panNETs) and sinistral portal hypertension (SPH) and provide insights into the pathogenesis.

Methods: A retrospective review of panNETs was conducted from our institution for 12 years. Medical imaging findings were analyzed to determine any association with splenic vein thrombosis (SVT) at diagnosis. The cases were further selected based on the criteria for SPH, namely, (1) presence of SVT, (2) gastric varices, (3) patent portal vein, and (4) normal liver function tests.

Results: There were 61 patients with panNETs and 8 (8/61) had SVT and gastric varices at diagnosis. Four (4/8) met the strict criteria for SPH while the other four had more conventional portal hypertension. The four with SPH had large tumors located in the tail with splenic vein invasion and three of four presented with bleeding gastric varices. All four patients underwent surgical resection. Mean follow-up was 8.5 years and the hematemesis never recurred. The other four patients (four of eight) with gastric varices had unresectable disease and all died after a mean survival of 29 months.

Conclusion: PanNETs appear to be more commonly associated with SVT and SPH compared with other tumors. This could be related to their relatively indolent nature and their intrinsic vascularity. From a surgical viewpoint, the decision to operate depends on many factors including but not limited to the size/stage, grade, and functionality of the tumor and comorbidities. These considerations notwithstanding, the association between panNETs and SPH suggests that there is benefit in timely resection of panNETs located in the tail.
\end{abstract}

Keywords: gastric varices; pancreatic neuroendocrine tumors; pathogenesis; sinistral portal hypertension; splenic vein thrombosis

\section{Introduction}

Sinistral portal hypertension (SPH) is a localized or leftsided form of extrahepatic portal hypertension that may lead to gastric variceal hemorrhage. The pathogenesis of $\mathrm{SPH}$ is related to splenic vein thrombosis (SVT), which causes retrograde blood flow through the short and posterior gastric and gastroepiploic veins. ${ }^{1-4}$ In contrast to conventional portal hypertension, the features of SPH occur in the absence of chronic liver disease.
The most common causes of SPH are chronic pancreatitis, pseudocysts, and pancreatic tumors. ${ }^{1-8}$ In the case of chronic pancreatitis, the mechanism relates to fibrosis with resultant compression of the splenic vein. For pancreatic neoplasms, the most common tumor is ductal adenocarcinoma. It is an aggressive malignancy and the mechanism for SPH in this case is direct invasion or encasement of the vessels by the tumor. Since this tumor has such a dismal prognosis with a mean survival

Departments of ${ }^{1}$ Pathology and Lab Medicine and ${ }^{2}$ Medical Imaging, The Ottawa Hospital and University of Ottawa, Ottawa, Ontario, Canada.

${ }^{3}$ Division of Hepatobiliary Surgery, The Ottawa Hospital and University of Ottawa, Ottawa, Canada.

*Address correspondence to: Terence N. Moyana, MD, Department of Pathology and Laboratory Medicine, The Ottawa Hospital \& University of Ottawa, 501 Smyth Road, Ottawa, ON K2R 1A8, Canada, E-mail: tmoyana@ottawahospital.on.ca

(c) Terence N. Moyana et al. 2017; Published by Mary Ann Liebert, Inc. This is an Open Access article distributed under the terms of the Creative Commons Attribution License, which permits unrestricted use, distribution, and reproduction in any medium, provided the original work is properly cited. 
of $<12$ months, ${ }^{9,10} \mathrm{SPH}$ is generally a lesser consideration in the management of these patients. Conversely, pancreatic neuroendocrine tumors (panNETs), as a group, are relatively indolent tumors and the patients can live for many years even with metastatic disease. ${ }^{9-11}$

A review of the literature shows that most studies on SPH and pancreatic tumors are case reports or small series. ${ }^{12-21}$ However, an analysis of these studies suggests that panNETs are disproportionately cited with regard to SPH compared with other benign or lowgrade pancreatic tumors. In this report, we reviewed panNETs focusing on those complicated by SVT and $\mathrm{SPH}$, and we were interested in ascertaining the mechanistic basis for this association.

\section{Methods}

Patient selection

The study received approval from our institutional review board. The records of our pathology department were searched for all cases of panNETs between 2002 and 2016. More detailed information about the clinical history, medical imaging, laboratory findings, patient management, and follow-up was obtained from the hospital's electronic medical records.

\section{Pathology}

The diagnosis of panNET was based on routine hematoxylin and eosin slides. In select cases, additional stains such as Congo red and Masson trichrome were utilized. Immunohistochemical assessments of the neuroendocrine markers synaptophysin and chromogranin A were conducted on a representative block from the surgical biopsy, resection, or cytology specimens as previously described. ${ }^{22}$ In some cases, additional stains were used to further substantiate the immunohistochemical profile or diagnosis, for example, insulin, somatostatin, glucacon, gastrin, and serotonin.

\section{Medical imaging}

The medical imaging findings of the panNET patients were reviewed to determine whether any were associated with SVT at the time of diagnosis. All patients underwent computed tomography (CT) of the chest, abdomen, and pelvis with intravenous (IV) contrast enhancement. A few selected cases had magnetic resonance imaging or ultrasound depending on their clinical status. All the cases were performed with IV contrast and most with a dedicated multiphasic pancreatic cancer protocol. Case 1 (Fig. 1A) was scanned with pancreatic parenchymal phase and portal venous phase $(\mathrm{kV} 120, \mathrm{~mA} 280$, slice
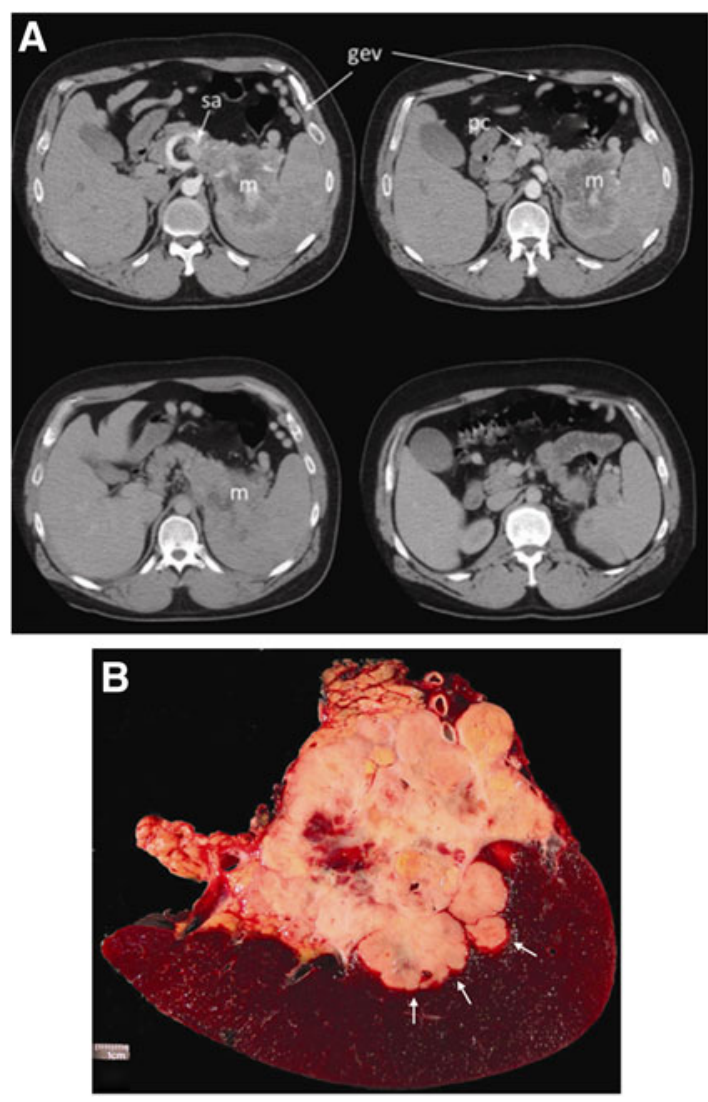

FIG. 1. (A) Case 1. CT with contrast in the pancreatic parenchymal phase (top row) and portal venous phase (bottom row). A large invasive $m$ arising from the tail of the pancreas extends into the hilum of the spleen. The axial plane images are analogous to the gross pathology (B). The direct invasion of the spleen from the pancreatic tail obstructs the splenic vein to the level of the pc. The sa is encased and remains patent. The splenic vein is obliterated and not visible on either phase. Large gev collateral shunts venous blood from the spleen back into the portal vein through collaterals in the pancreatic head. The dilated and enhancing gev is easily seen on $\mathrm{CT}$ running in a tortuous course inferior to the greater curvature of the stomach. (B) Case 1. Crosssection of pancreatic tail where the pancreatic neuroendocrine tumor is invading into the splenic parenchyma. Note the pushing margin of the tumor as bulbous nodules (arrows). Residual normal (lobulated) pancreatic tissue is seen right at the top (arrow). gev, gastroepiploic vein; $\mathrm{m}$, mass; pc, portal confluence; sa, splenic artery. 
thickness $2.5 \mathrm{~mm}$, and $5 \mathrm{~mm}$ on a GE Lightspeed Plus CT Unit [GE Healthcare, Milwaukee, WI]). The corresponding surgical resected specimen is shown in Figure 1B. Case 2 had a contrast-enhanced abdominal magnetic resonance (Fig. 2) with coronal and axial postgadolinium contrast T1-weighted Vibe FS images (repetition time [TR] 4.64, echo time [TE] 2.4, $240 \times 320 \mathrm{~mm}$ field of view, $3 \mathrm{~mm}$ slice thickness, on a Siemens 1.5T TRIO Unit [Siemen Medical Systems, Erlangen, Germany]) and axial T2-weighted turbo spin echo images (TR 3206, TE $81,240 \times 320 \mathrm{~mm}$ field of

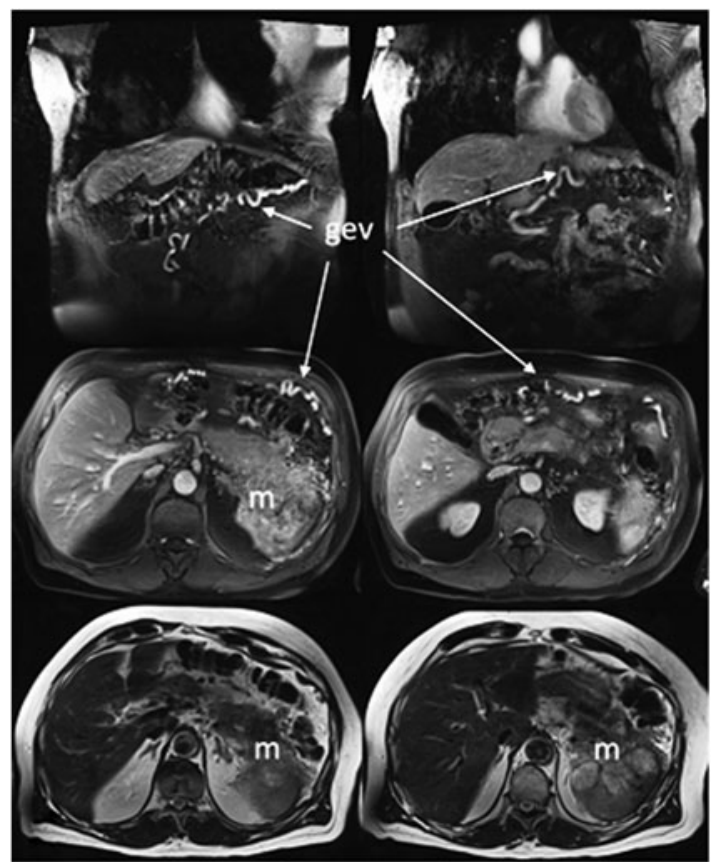

FIG. 2. Case 2. This figure shows coronal and axial MR image with fat saturation and contrast in the portal venous phase, and axial T2-weighted images (bottom row). A large invasive $m$ arising from the tail of the pancreas extends into the hilum of the spleen. The direct invasion of the spleen from the pancreatic tail invades and obstructs the splenic vein from the level of the pancreatic tail. The splenic vein is obliterated at the splenic hilum and not visible. A large gev shunts venous blood from the spleen back into the patent portal vein through collaterals in the pancreatic head. The dilated and enhancing gev is easily seen on postcontrast MR images running in a tortuous course inferior to the greater curvature of the stomach. MR, magnetic resonance. view, $5 \mathrm{~mm}$ slice thickness). Majority of the patients underwent Octreotide scan with SPECT CT (In-111 chelated to octreotide using diethylenetriaminepentaacetic acid [In-111 penetetreotide] as part of their work-up (images not shown).

\section{Results}

A total of 61 patients with panNETs were identified. Eight of them (8/61) were found to have SVT and gastric varices at the time of diagnosis (Table 1). Of these, four (four of eight; cases 1,2, 7, and 8) met the strict criteria for SPH as defined by the presence of SVT, gastric varices, patent portal vein, and normal liver function tests. ${ }^{1-6}$ Three of these patients presented with hematemesis from bleeding gastric varices. They were resuscitated and stabilized. Patient 8 presented with abdominal pain but without a variceal hemorrhage. After work-up, all four patients underwent distal pancreatectomy and splenectomy with partial gastrectomy. The tumors were large with a mean maximum dimension of $9.7 \mathrm{~cm}$ (Figs. 1 and 2). They were located in the tail (Fig. 3A,B) with extrapancreatic extension and invasion and thrombosis of the splenic vein (Fig. 4A,B).

Microscopy of all the eight cases with SVT showed that the tumors had characteristic features of panNETsan organoid morphology with some areas having a trabecular pattern, others being diffuse, gyriform, or nested (Fig. 3A,B). The cells had round to oval nuclei with variable pale to amphophilic cytoplasm. Mitotic activity was low, ranging from 1 to 3 per 10 highpower fields, except for case 3 that showed 5. In all the eight cases with SVT, the Congo red stain for amyloid was negative. By immunohistochemistry, the stains for insulin, somatostatin, glucagons, gastrin, and serotonin were negative. The Ki-67 labeling index was determined using the camera-captured/printed image method. ${ }^{23}$ Using the 2017 World Health Organization classification, seven of the eight cases were classified as welldifferentiated panNETs, grade $2,,^{24,25}$ and one as grade 3 (Table 1).

Patient 1 was staged as pT3N0M0 (American Joint Commission for Cancer). The surgical margins were clear but with narrow clearance. Since he was only 42 years old and the tumor was relatively large with narrow margins, he was given adjuvant radiotherapy to the tumor bed to reduce the possibility of local recurrence. The gastric varices never recurred, but 2 years postsurgery he developed liver metastases that were managed by radiofrequency ablation and chemotherapy. He did well as his disease was kept under control 


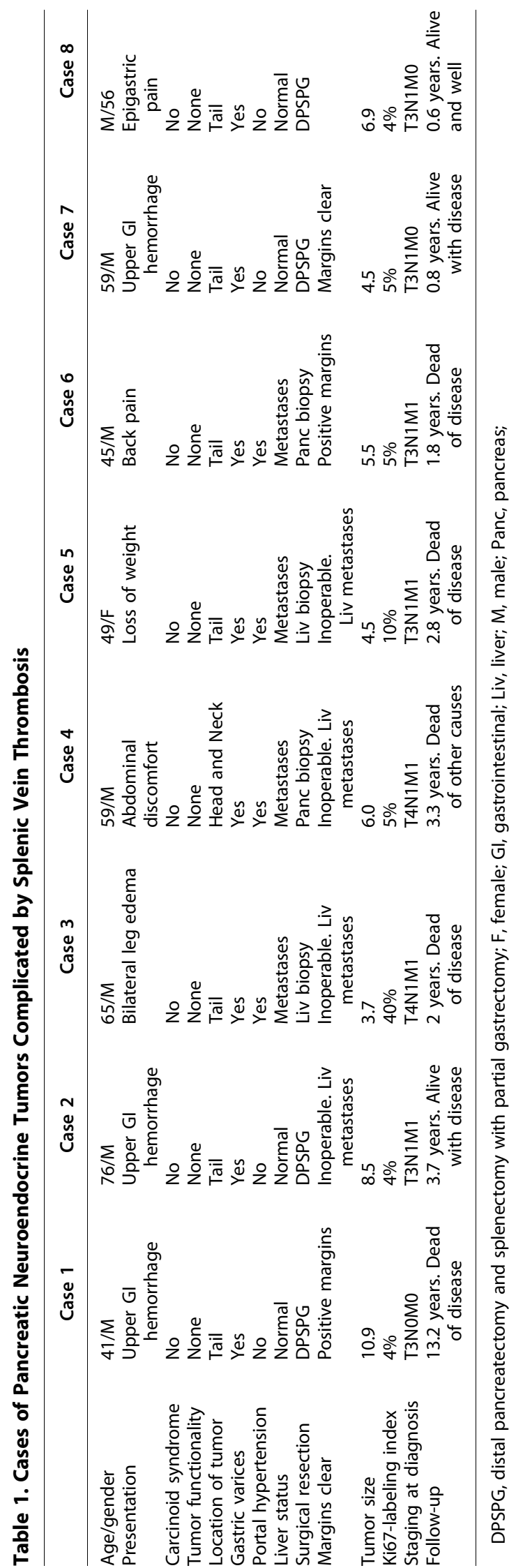

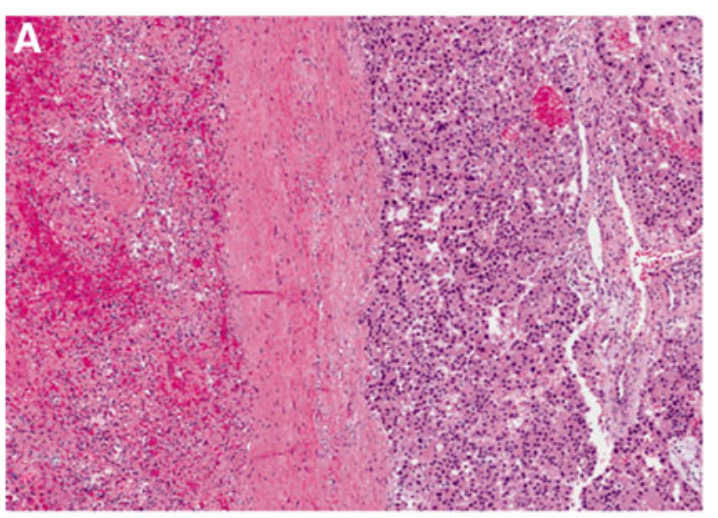

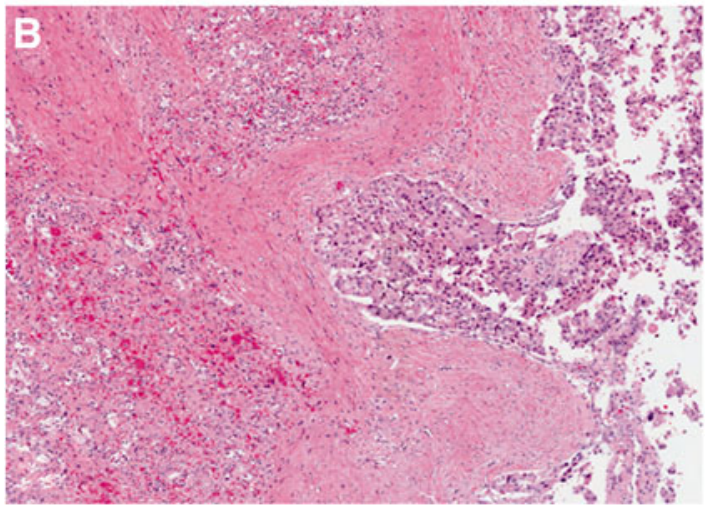

FIG. 3. (A) Case 2. Photomicrograph showing tumor on right abutting splenic capsule (longitudinal fibrous band) with splenic parenchyma on left (hematoxylin eosin). (B) Case 2. Photomicrograph depicting tumor on right invading into splenic capsule as a broad pushing front with splenic parenchyma on left (hematoxylin eosin).

for many years. He eventually succumbed to metastases 13.2 years after his surgery.

For patient 2, based on preoperative imaging, it was determined that curative resection was unlikely; however, surgery was undertaken with the intent of preventing further variceal bleeding. The pathology specimen was staged as pT3N1M1 with the M1 being attributed to a separate wedge resection of diaphragm that was positive for tumor. He also had positive surgical margins. He was treated with postoperative chemotherapy. Approximately 1 year after surgery, he developed small volume metastatic liver disease that appears to have been controlled by chemotherapy and thus far has been nonprogressive. He is now $>3$ years postsurgery, and the varices have not recurred. 


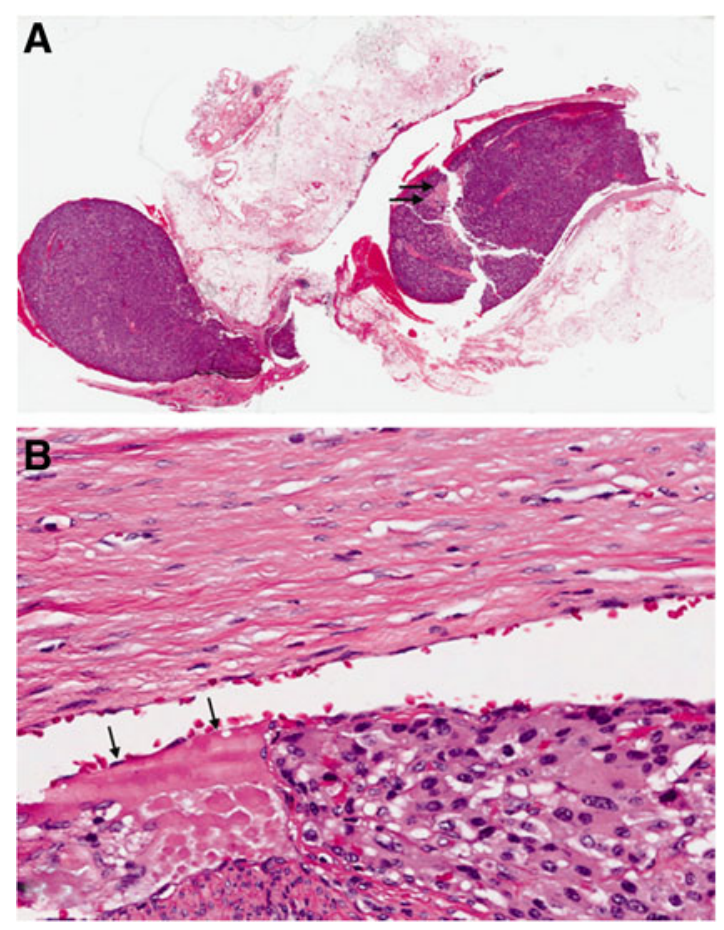

FIG. 4. (A) Case 2. Photomicrograph with the tumor having invaded and distending the splenic vein. The lightly eosinophilic areas (arrow) show early thrombus formation (hematoxylin eosin). (B) Case 2. Higher magnification of tumor within splenic vein highlighting the thrombosis (arrow) (hematoxylin eosin).

Patient 7 had varices that were glued at endoscopy. He then underwent surgery and was found to have positive margins postresection. Owing to a concurrent psychiatric illness and other comorbidities, he was not given any postoperative chemo- or radiotherapy. One year after resection, the varices have not recurred and the disease has not progressed. He remains under watchful observation.

Patient 8 presented with abdominal pain and was found to have a pancreatic mass. It was resected with clear margins. Eight months later, he is alive and well with no evidence of disease or varices.

The other four patients (four of eight) with SVT and gastric varices (patients 3-6) already had liver metastases with portal hypertension and/or portal vein thrombosis. All these four patients had unresectable disease and were mainly treated with chemotherapy. They died of disease or other causes with a mean survival of 29 months.

\section{Discussion}

This is a retrospective review of 61 patients with panNETs for a 12-year period. Eight of these patients were found to have SVT and gastric varices but only four fulfilled the strict criteria for $\mathrm{SPH} .{ }^{1-6}$ In all four patients, the tumors were relatively large, located in the tail with extrapancreatic extension and invasion of the splenic vein. Even though there were no liver metastases, the extensiveness of the locoregional disease augered against a curative resection. However, since three of these patients had presented with life-threatening hematemesis, surgical resection was necessary irrespective of whether it was curative or debulking/palliative. It turned out that in one case, there was complete surgical clearance although with narrow margins, whereas the other two cases had positive margins. It is noteworthy that the varices and hematemesis never recurred after a mean of 54.9 months postsurgery. The surgical resection and splenectomy decompress the splanchnic venous pressure in this region, which effectively removes a life-threatening complication and thus prolongs survival and quality of life. ${ }^{1-8}$

SPH can occur in various conditions, both nonneoplastic and neoplastic. The most common nonneoplastic association is with chronic pancreatitis and the mechanism here relates to fibrosis. ${ }^{1-8}$ In those cases of pancreatitis complicated by pseudocyst formation, the pseudocyst itself can cause compression of splenic vein. ${ }^{1-4,12}$ The neoplastic associations include not only pancreatic tumors such as panNETs, ${ }^{11-21}$ solid pseudopapillary neoplasms, ${ }^{26}$ mucinous ${ }^{27}$ or serous cystadenomas, ${ }^{28}$ and ductal adenocarcinoma ${ }^{2,6}$ but also nonpancreatic neoplasms, for example, renal cell carcinoma ${ }^{29}$ and colonic lymphoma, ${ }^{30}$ and even ectopic spleen. ${ }^{31}$ Nonetheless, pancreatic tumors, particularly panNETs tumors, ${ }^{11-21}$ are more commonly implicated than others. In this report, we document additional cases of panNETs that were complicated by SPH and upper gastrointestinal hemorrhage.

The fact that panNETs $(\sim 1-2 \%$ of pancreatic tumors $)^{9-11}$ are more commonly cited in relation to $\mathrm{SPH}$ than ductal adenocarcinomas (85\% of pancreatic tumors $)^{9,10}$ or any other tumors for that matter suggests that the pathogenesis of SPH may not simply be based on the invasion or compression by tumor. It could be due to the secretion of fibrogenic neurohormonal substances in much the same way that small bowel NETs secrete serotonin and other biogenic amines that cause mesenteric fibrosis, kinking of the bowel, vascular sclerosis, ischemia, and tricuspid stenosis. ${ }^{24,32,33}$ However, our patients did not manifest the carcinoid or other 
functional neurohormonal syndromes, and the tumors were negative for serotonin, insulin, somatostatin, and glucagon.

Conventional portal hypertension ${ }^{34}$ has been associated with hepatic amyloidosis. ${ }^{35}$ Since panNETs can produce amyloid $^{36,37}$ (Apud amyloid), we were interested in whether this could be a factor in the pathogenesis of SVT and SPH. However, all eight of our cases with SVT were negative for amyloid. We, therefore, hypothesize that the predisposition of panNETs to SVT and SPH may simply be related to the intrinsic vascularity ${ }^{38}$ of these tumors just like other NETs in general. In the face of SVT, such vascularity could exacerbate blood flow and backpressure on short gastric and gastroepiploic veins.

Despite recent advances in the medical management of panNETs with newer drugs such as octreotide, sunitinib, and everolimus, ${ }^{39,40}$ surgery remains the mainstay of treatment. That being said, many considerations have to be factored into the decision to operate. They include but are not limited to the size/stage, grade, and functionality of the tumor and surgical comorbidities. ${ }^{41-44}$ These considerations notwithstanding, our study suggests that there is benefit to timely resection of panNETs located in the tail. Furthermore, even in patients wherein a curative resection is not possible, palliative resection with splenectomy can improve the quality of life and outcomes.

In summary, it would appear that panNETs are more likely to get complicated by SVT and SPH than other pancreatic neoplasms. This may be related to their relatively indolent nature and intrinsic vascularity. Although this proposed hypothesis is more germane to the four cases that strictly meet the criteria of SPH, mechanistically it could to some extent also apply to the other four panNETs with SVT and conventional portal hypertension.

\section{Author Disclosure Statement}

No competing financial interests exist.

\section{References}

1. Sakorafas GH, Sarr MG, Farley DR, et al. The significance of sinistral portal hypertension complicating chronic pancreatitis. Am J Surg. 2000;179:129-133.

2. Thompson RJ, Taylor MA, McKie LD, et al. Sinistral portal hypertension. Ulster Med J. 2006;75:175-177.

3. Agarwal AK, Raj Kumar K, Agarwal S, et al. Significance of splenic vein thrombosis in chronic pancreatitis. Am J Surg. 2008;196:149-154.

4. Köklü S, Coban S, Yüksel O, et al. Left-sided portal hypertension. Dig Dis Sci. 2007;52:1141-1149.

5. Heider TR, Azeem S, Galanko JA, et al. The natural history of pancreatitisinduced splenic vein thrombosis. Ann Surg. 2004;239:876-880-882.

6. Chang CY. Pancreatic adenocarcinoma presenting as sinistral portal hypertension: an unusual presentation of pancreatic cancer. Yale J Biol Med. 1999;72:295-300.
7. Smith TA, Brand EJ. Pancreatic cancer presenting as bleeding gastric varices. J Clin Gastroenterol. 2001;32:444-447.

8. Hwang $T L$, Jan $Y Y$, Jeng $L B$, et al. The different manifestation and outcome between pancreatitis and pancreatic malignancy with left-sided portal hypertension. Int Surg. 1999;84:209-212.

9. Reid MD, Bagci P, Adsay NV. Histopathologic assessment of pancreatic cancer: does one size fit all? J Surg Oncol. 2013;107:67-77.

10. Fesinmeyer MD, Austin MA, $\mathrm{Li} \mathrm{Cl}$, et al. Differences in survival by histologic type of pancreatic cancer. Cancer Epidemiol Biomark. 2005;14:17661773.

11. Vagefi PA, Razo O, Deshpande V, et al. Evolving patterns in the detection and outcomes of pancreatic neuroendocrine neoplasms: the Massachusetts General Hospital experience from 1977 to 2005. Arch Surg. 2007;142:347-354.

12. Evans GR, Yellin AE, Weaver FA, et al. Sinistral (left-sided) portal hypertension. Am Surg. 1990;56:758-763.

13. Wong S-W, Lee K-F, Lai PB-S. Pancreatic neuroendocrine tumour presented as isolated gastric varices. Can J Surg 2007;50:143-144.

14. Metz DC, Benjamin SB. Islet cell carcinoma of the pancreas presenting as bleeding from isolated gastric varices. Report of a case and review of the literature. Dig Dis Sci. 1991;36:241-244.

15. Yamaguchi T, Takahashi $\mathrm{H}$, Kagawa $\mathrm{R}$, et al. Nonfunctioning pancreatic endocrine tumor presenting with hemorrhage from isolated gastric varices. Am Surg. 2005;71:1027-1030.

16. Joyce DL, Hong K, Fishman EK, et al. Multi-visceral resection of pancreatic VIPoma in a patient with sinistral portal hypertension. World J Surg Oncol. 2008;6:80.

17. Rodriguez RA, Overton $H$, Morris KT. Pancreatic neuroendocrine tumor with splenic vein tumor thrombus: a case report. Int J Surg Case Rep. 2014;5:1271-1274.

18. Olakowski $M$, Lampe $P$, Bołdys $H$, et al. Neuroendocrine pancreatic carcinoma causing sinistral portal hypertension. Med Sci Monit. 2001;7: 1326-1328.

19. Wang $\mathrm{H}$, Bie $\mathrm{P}$, Zhang L. Refractory gastroesophageal variceal bleeding secondary to neuroendocrine carcinoma in the pancreatic tail. Pancreatology. 2011;11:228-232.

20. Dumont F, Goudard Y, Caramella C, et al. Therapeutic strategies for advanced pancreatic neuroendocrine tumors with segmental portal hypertension. World J Surg. 2015;39:1974-1980.

21. Huang S-H, Wu S-Y, Chang Y-M, et al. Endocrine carcinoma of the pancreatic tail exhibiting gastric variceal bleeding. J Med Sci. 2014;34:217.

22. Moyana TN, Kendal WS, Chatterjee A, et al. Role of fine-needle aspiration in the surgical management of pancreatic neuroendocrine tumors: utility and limitations in light of the new World Health Organization classification. Arch Pathol Lab Med. 2014;138:896-902.

23. Reid MD, Bagci P, Ohike N, et al. Calculation of the Ki67 index in pancreatic neuroendocrine tumors: a comparative analysis of four counting methodologies. Mod Pathol. 2015;28:686-694.

24. Klimstra DS, Yang Z. Pathology, classification, and grading of neuroendocrine tumors arising in the digestive system. In: UpToDate. Goldberg RM (ed.) UpToDate: Waltham, MA. Available at: www.uptodate.com/ contents/pathology-classification-and-grading-of-neuroendocrine-tumorsarising-in-the-digestive-system Accessed August 9, 2015.

25. Reid MD, Balci S, Saka B, et al. Neuroendocrine tumors of the pancreas: current concepts and controversies. Endocr Pathol. 2014;25:65-79.

26. Nakamura S, Takayama Y, Kuboki Y, et al. A case of solid pseudopapillary neoplasm of the pancreas presenting with left-sided extrahepatic portal hypertension. Intern Med Tokyo Jpn. 2010;49:1749-1753.

27. Thrainsdottir $\mathrm{H}$, Petursdottir V, Blöndal S, et al. Pancreatic mass leading to left-sided portal hypertension, causing bleeding from isolated gastric varices. Case Rep Gastrointest Med. 2014;2014:956490.

28. Ito K, Kudo A, Nakamura N, et al. Left-sided portal hypertension caused by serous cystadenoma of the pancreas: report of a case. Surg Today. 2008;38:184-187.

29. Joya Seijo MD, del Valle Loarte P, Marco Martínez J, et al. [Sinistral portal hypertension with bleeding gastric varices as initial manifestation of renal-cell carcinoma]. An Med Interna. 2004;21:283-284.

30. Seenu V, Goel AK, Shukla NK, et al. Hodgkin's lymphoma of colon: an unusual cause of isolated splenic vein obstruction. Indian J Gastroenterol. 1994;13:70-71.

31. Angerås $U$, Almskog $B$, Lukes $P$, et al. Acute gastric hemorrhage secondary to wandering spleen. Dig Dis Sci. 1984;29:1159-1163. 
32. Bhattacharyya S, Toumpanakis C, Chilkunda D, et al. Risk factors for the development and progression of carcinoid heart disease. Am J Cardiol. 2011;107:1221-1226.

33. Washington MK, Tang LH, Berlin J, et al. Protocol for the examination of specimens from patients with neuroendocrine tumors (carcinoid tumors) of the small intestine and ampulla. Arch Pathol Lab Med. 2010;134:181-186.

34. Al-Osaimi AM, Caldwell SH. Medical and endoscopic management of gastric varices. Semin Intervent Radiol. 2011;28:273-282.

35. Krasinskas AM, Eghtesad B, Kamath PS, et al. Liver transplantation for severe intrahepatic noncirrhotic portal hypertension. Liver Transplant. 2005;11:627-634-611.

36. Frankel WL. Update on pancreatic endocrine tumors. Arch Pathol Lab Med. 2006;130:963-966.

37. Asa SL. Pancreatic endocrine tumors. Mod Pathol 2011;24(Suppl 2):S66S77.

38. Tan G, Cioc AM, Perez-Montiel D, et al. Microvascular density does not correlate with histopathology and outcome in neuroendocrine tumors of the pancreas. Appl Immunohistochem Mol Morphol 2004;12:31-35.

39. Kunz PL, Reidy-Lagunes D, Anthony LB, et al. Consensus guidelines for the management and treatment of neuroendocrine tumors. Pancreas. 2013;42:557-577

40. Strosberg JR, Fine RL, Choi J, et al. First-line chemotherapy with capecitabine and temozolomide in patients with metastatic pancreatic endocrine carcinomas. Cancer. 2011;117:268-275.

41. Kuo EJ, Salem RR. Population-level analysis of pancreatic neuroendocrine tumors $2 \mathrm{~cm}$ or less in size. Ann Surg Oncol. 2013;20:2815.

42. Bettini R, Partelli $S$, Boninsegna $L$, et al. Tumor size correlates with malignancy in non-functioning pancreatic endocrine tumor. Surgery. 2011;150:75-82.
43. Regenet N, Carrere N, Boulanger $\mathrm{G}$, et al. Is the 2-cm size cutoff relevant for small non-functioning pancreatic neuroendocrine tumors. A French multicenter study. Surgery. 2016;159:901-907.

44. Rosenberg AM, Friedmann P, Del Rivero J. Resection versus expectant management of small incidentally discovered non-functional neuroendocrine tumors. Surgery. 2016;159:302-310.

Cite this article as: Moyana TN, Macdonald DB, Martel G, Pyatibrat S, Lee G, Capitano M (2017) Pancreatic neuroendocrine tumors complicated by sinistral portal hypertension: insights into pathogenesis, Journal of Pancreatic Cancer 3:1, 71-77, DOI: 10.1089/pancan .2017.0017.

breviations Used

$\mathrm{CT}=$ computed tomography

DPSPG = distal pancreatectomy and splenectomy with partial gastrectomy

$\mathrm{Gl}=$ gastrointestina

$\mathrm{IV}=$ intravenous

$\mathrm{MR}=$ magnetic resonance

panNETs = pancreatic neuroendocrine tumors

$\mathrm{SPH}=$ sinistral portal hypertension

$\mathrm{SVT}=$ splenic vein thrombosis

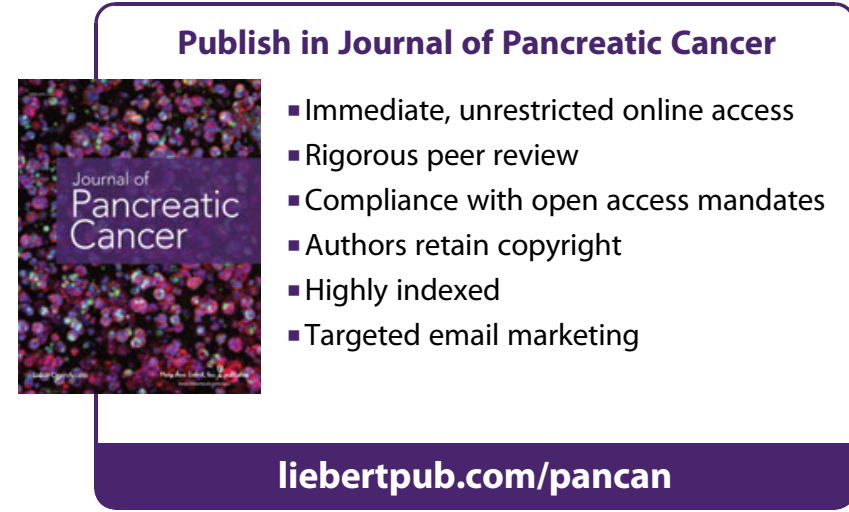

